\title{
THE RENEWAL PROCESS GENERATED BY RETURN TIMES OF SEMI-MARKOV PROCESS IN RELIABILITY MODELS
}

\section{PROCES ODNOWY GENEROWANY PRZEZ CZASY POWROTU PROCESU SEMI-MARKOWSKIEGO W MODELACH NIEZAWODNOŚCI}

\begin{abstract}
Franciszek Grabski
Akademia Marynarki Wojennej, Polish Naval Academy

Abstract. The renewal process generated by the return times of semi-Markov process to a given state is considered in the paper. The return time to a state $j$ and also a first passage time from a given state $i$ to the state $j$ of semi-Markov process are basic concepts that are used to determine this process. The systems of equations for distributions, expectations and secondond moments of these random variables are presented. Theorem concerning the asymptotic distribution of the considered renewal process is presented in this article. Moreover an illustrative example from the reliability theory is presented in the paper.

Keywords: Renewal process, semi-Markov model,

Streszczenie: $W$ artykule jest rozważany proces odnowy generowany przez czasy powrotu procesu semi-Markowa (SM) do danego stanu. Czas powrotu do stanu j, a także czas pierwszego przejście od danego stanu $i$ do stanu j semi-Markowa procesu sa podstawowymi pojęciami, które sa stosowane do określenia tego procesu. W pracy zostaly przedstawione układy równań dla transformat rozkładów, wartości oczekiwanych i drugich momentów tych zmiennych losowych. Twierdzenie dotyczace asymptotycznego rozkladu badanego procesu odnowy jest kluczowym elementem pracy. Ponadto zostat przestawiony przyktad z teorii niezawodności ilustrujący rozważane problemy.
\end{abstract}

Stowa kluczowe: Proces odnowy, proces semi-Markowski 
The renewal process generated by return times of semi-Markov process in...

Proces odnowy generowany przez czasy powrotu procesu semi-Markowskiego...

\section{THE RENEWAL PROCESS GENERATED BY RETURN TIMES OF SEMI-MARKOV PROCESS IN RELIABILITY MODELS}

\section{Introduction}

One of the quantities connected to the semi-Markov (SM) process are a return time to a state $j$ and a first passage time from given state $i$ to the state $j$. These quantities are random variables, the distribution of which can be calculated on the basis of the kernel of SM process. Consecutive return times and the first passage time allow to determine a general renewal process, the value of which at the moment $t$ denotes a number of inputs to the state $\mathrm{j}$ in a time interval $[0, t]$. Combining results from the semi-Markov processes theory and renewal theory we can obtain equations for distribution and parameters of the process and its approximation. Moreover one illustrative example of the reliability problem is presented in the paper.

\section{Necessary definitions and theorems}

Suppose that $\{X(t): t \geq 0\}$ is a semi-Markov process determined by the continuous kernel $Q(t), t \geq 0$, where $S$ is discrcrete state space. Assume that a state $j \in S$ is strongly recurrent and strongly accessible from the state $i \in S$ [3]. Consider the sequence of random variables

$$
\Theta_{i j}^{(1)}, \Theta_{j j}^{(2)}, \Theta_{j j}^{(3)}, \ldots
$$

Where $\Theta_{i j}^{(1)}=\Theta_{i j}$ represents a first passage from the state $i$ to the state $j$ while random variables $\Theta_{j j}^{(n)}, n=2,3, \ldots$ form the sequence of return time to the state $j \in S$. The random variable $\Theta_{i j}^{(1)}$ has cumulalive distribution function (CDF) ma

$$
\Phi_{i j}(t)=P\left\{\Theta_{i j}^{(1)} \leq t\right\} .
$$

It is proved [ 3 ], that $\mathrm{CDF} \Phi_{i j}(t), i, j \in \mathrm{S}$, under assumption which are satisfied in cinsidered model, are the only solutions of the equations system that Laplace Stieltjes transformation takes the form

$$
\tilde{\phi}_{i A}(s)=\sum_{j \in A} \tilde{q}_{i j}(s)+\sum_{k \in A^{\prime}} \tilde{q}_{i k}(s) \tilde{\phi}_{k A}(s), i \in A^{\prime} .
$$

where

$$
\tilde{\phi}_{i A}(s)=\int_{0}^{\infty} e^{-s t} d \Phi_{i A}(t), \quad \tilde{q}_{i j}(s)=\int_{0}^{\infty} e^{-s t} d Q_{i j}(t) .
$$


We have obtained a linear system of equations, where coefficients are transforms $\tilde{q}_{i j}(s)$ and the unknowns are transforms $\tilde{\phi}_{i A}(s)$. The linear system of equations is equivalent to the matrix equation

$$
\left(I-q_{A^{\prime}}(s)\right) \phi_{A^{\prime}}(s)=b(s),
$$

where $I=\left[\delta_{i j}: i, j \in A^{\prime}\right] \quad$ is the unit matrix, $q_{A^{\prime}}(s)=\left[\tilde{q}_{i j}(s): i, j \in A^{\prime}\right]$ is the square submatrix of the L-S transform of the matrix $q(s)$, while $\phi_{A^{\prime}}(s)=\left[\tilde{\phi}_{i A}(s): i \in A^{\prime}\right]^{T}, \quad b(s)=\left[\sum_{j \in A} \tilde{q}_{i j}(s): i \in A^{\prime}\right]^{T} \quad$ are one-column matrices of the corresponding L-S transforms. The equation allows us to obtain the equations for the moments of random $\Theta_{i A}, i \in A^{\prime}$.

The expectations $E\left(\Theta_{i A}\right), i \in A^{\prime}$ are unique solution of the linear equations system, which have the following matrix form

where

$$
\left(I-P_{A^{\prime}}\right) \bar{\Theta}_{A^{\prime}}=\bar{T}_{A^{\prime}}
$$

$$
P_{A^{\prime}}=\left[p_{i j}: i, j \in A^{\prime}\right], \bar{\Theta}_{A^{\prime}}=\left[E\left(\Theta_{i A}\right): i \in A^{\prime}\right]^{T}, \quad \bar{T}_{A^{\prime}}=\left[E\left(T_{i}\right): i \in A^{\prime}\right],
$$

and $I$ is the unit matrix.

The second moments $E\left(\Theta_{i A}\right), i \in A^{\prime}$ are unique solution of the linear equations system, which have the following matrix form

$$
\begin{gathered}
\left(I-P_{A^{\prime}}\right) \bar{\Theta}_{A^{\prime}}^{2}=B_{A}, \\
P_{A^{\prime}}=\left[p_{i j}: i, j \in A^{\prime}\right], \quad \bar{\Theta}_{A^{\prime}}=\left[E\left(\Theta_{i A}^{2}\right): i \in A^{\prime}\right]^{T}, \quad B_{A}=\left[b_{i A}: i \in A^{\prime}\right]^{T}, \\
b_{i A}=E\left(T_{i}^{2}\right)+2 \sum_{k \in A^{\prime}} p_{i k} E\left(T_{i k}\right) E\left(\Theta_{k A}\right) .
\end{gathered}
$$

The random variables $\Theta_{j j}^{(n)}, \ldots, n=2,3, \ldots$ denote the consecutive return times to the state $j$ and

is $\mathrm{CDF}$ of them.

$$
\Phi_{j j}(t)=P\left\{\Theta_{j j}^{(n)} \leq t\right\}=P\left\{\Theta_{j j} \leq t\right\}
$$

The equation for the Laplace-Stieltjes trnsform has the form

$$
\tilde{\phi}_{j j}(s)=\tilde{q}_{j j}(s)+\sum_{k \in S-\{j\}} \tilde{q}_{j k}(s) \tilde{\phi}_{k j}(s),
$$

and the equations for expectation and second moment are

$$
E\left(\Theta_{j j}\right)=E\left(T_{j}\right)+\sum_{k \in S-\{j\}} p_{j k} E\left(\Theta_{k j}\right),
$$


The renewal process generated by return times of semi-Markov process in... Proces odnowy generowany przez czasy powrotu procesu semi-Markowskiego...

$$
E\left(\Theta_{j j}^{2}\right)=E\left(T_{j}^{2}\right)+\sum_{k \in S-\{j\}} p_{j k} E\left(\Theta_{k j}^{2}\right)+2 \sum_{k \in S-\{j\}} p_{j k} E\left(T_{j k}\right) E\left(\Theta_{k j}\right) .
$$

It can be shown that the random variables $\Theta_{i j}^{(1)}, \Theta_{j j}^{(2)}, \ldots, \Theta_{j j}^{(n)}, \quad n=2,3, \ldots$ are mutually independent.

Let

$$
S_{i j}^{(n)}=\Theta_{i j}^{(1)}+\Theta_{j j}^{(2)}+\cdots+\Theta_{j j}^{(n)}, \quad n=2,3, \ldots
$$

and

$$
V_{i j}(t)=\sup \left\{n: S_{i j}^{(n)} \leq t\right\}, \quad t \geq 0,
$$

The value of a random variable $V_{i j}(t)$ denotes the number of visits of the SM procecess in the state $j \in S$ in the time interval $[0, t]$ if the initial state is $i$. The stochastic process $\left\{V_{i j}(t): t \geq 0\right\}$ is called the renewal process genereted by the first passage time and return times of the $S M$.

Adopting theorems of the renewal theory we can formulate a limit theorem concerning the stochastic $\left\{V_{i j}(t): t \geq 0\right\}$ as $t \rightarrow \infty$.

Theorem [ 2 ], [ 3 ]. If $j$ is a positive recurrent state, $j$ is strongly accessible from the state $i \in S$, the random variables that are independent copies of the random variable $\Theta_{j j}$ have the finite positive expected values and variances, then

$$
\lim _{t \rightarrow \infty} P\left(\frac{V_{i j}(t)-\frac{t-E\left(\Theta_{i j}\right)+E\left(\Theta_{j j}\right)}{E\left(\Theta_{j j}\right)}}{\sqrt{\frac{V\left(\Theta_{j j}\right) t}{\left[E\left(\Theta_{j j}\right)\right]^{3}}}} \leq x\right)=\frac{1}{\sqrt{2 \pi}} \int_{-\infty}^{x} e^{\frac{u^{2}}{2}} d u .
$$

From this theorem it follows that for large $t$ the random variable $V_{i j}(t)$ is approximately normally distributed $N(m(t), \sigma(t))$ with the expected value $m(t)$ and standard deviation $\sigma(t)$ :

$$
m(t)=\frac{t-E\left(\Theta_{i j}\right)+E\left(\Theta_{j j}\right)}{E\left(\Theta_{j j}\right)}, \sigma(t)=\sqrt{\frac{D^{2}\left(\Theta_{j j}\right) t}{\left[E\left(\Theta_{j j}\right)\right]^{3}}} .
$$

The parameters $E\left(\Theta_{i j}\right), E\left(\Theta_{j j}\right), E\left(\Theta_{j j}^{2}\right)$ can be calculated easily on the basis of the equations (3), (5), (6). The variance is calculated using a well-known formula

$$
D^{2}(\Theta)=E\left(\Theta_{j j}^{2}\right)-\left[E\left(\Theta_{j j}\right)\right]^{2}
$$




\section{SM-model of renewable series system}

We will present semi-Markov model of a renewal system consisting of $k$ component that form a series reliability structure. That kind of system was modeled and studied in [1], [2], [3]. In this paper we will discuss one of the reliability characteristics of the system - number of failures in the time interval $[0, t]$.

\section{Description and assumptions}

A system consists of $n$ components which form a series reliability structure. We assume that a lifetime of component $k, k=1,2, \ldots, r$ is represented by a random variable $\zeta_{k}$ with exponential PDF

$$
f_{k}(t)=\lambda e^{-\lambda_{k} t} I_{[0, \infty)}(t) .
$$

From the structure of the system it follows that the damage of the system takes place if a failure of any component occurs. A damaged component is renewed. We assume that the renewal time of $k$-th component is a non-negative random variable $\gamma_{k}$ with a CDF

$$
H_{k}(t)=P\left(\gamma_{k} \leq t\right), \quad k=1,2, \ldots, r .
$$

We know that the exponential probability distribution has memoryless property. Therefore the renewal of component means renewal of the whole system. We also assume that the random variables denoting successive times to failure of $k$-th component and random variables denoting their consecutive renewal times are independent copies of the random variables $\zeta_{k}$ and $\gamma_{k}$ accordingly. We suppose that the random variables $\zeta_{1}, \zeta_{2}, \ldots, \zeta_{n}, \gamma_{1}, \gamma_{2}, \ldots, \gamma_{n}$ are mutually independent. Moreover we assume that $\gamma_{1}, \gamma_{2}, \ldots, \gamma_{n}$ have the positive, finite expected values and variances.

\section{Model}

We introduce the following states:

$$
\begin{aligned}
& r+1 \text { - work of the ,up" system, } \\
& k \text { - renewal of a component } k, k=1, \ldots r \text { after its failure }
\end{aligned}
$$

The "down" states are represented by a set $A=\{1,2, \ldots, r\}$, while the „up" state is represented by one element set $A^{\prime}=\{r+1\}$.

Let $0=\tau_{0}<\tau_{1}<\cdots<\tau_{n} \cdots$ denote moments of the system state changes. These moments denote the instants of the system failures or the instants of a sytem work beginning.

From the assumptions it follows that a state of the system at the moment $\tau_{n+1}$ and the duration of the state that was achived at the moment $\tau_{n}$ does not depend on the states at the moments $\tau_{0}, \tau_{1}, \ldots, \tau_{n-1} \ldots$ and their duration. Therefore the stochastic process $\{X(t): t \geq 0\}$ determines by the rule

$$
X(t)=k \quad \text { for } \quad k \in\left[\tau_{n}, \tau_{n+1}\right), \quad k \in S=\{1, \ldots, r, r+1\}
$$

is semi-Markov process. 
The renewal process generated by return times of semi-Markov process in...

Proces odnowy generowany przez czasy powrotu procesu semi-Markowskiego...

From the description of the system functioning follows shape of the SM kernel.

$$
Q(t)=\left[\begin{array}{llll}
0 & 0 & \cdots & Q_{1 r+1}(t) \\
0 & 0 & \cdots & Q_{2 r+1}(t) \\
\cdots & & & \\
0 & 0 & \cdots & Q_{r r+1}(t) \\
Q_{r+11}(t) & Q_{r+12}(t) & \cdots & 0
\end{array}\right] .
$$

The model will be constructed if we determine all elements of SM kernel. First we define the elements of the last row of the matrix $Q(t)$. Change of the state $r+1$ to $k,(k=1, \ldots, r)$ in a time not greater than $t$ holds if and only if the event

occurs.

$$
\left\{0 \leq \zeta_{k} \leq t, \zeta_{i}>\zeta_{k} \geq 0, \quad i=1, \ldots, k-1, k+1, \ldots r\right\}
$$

Hence

where

$$
\begin{aligned}
Q_{r+1, k}(t) & =P\left\{\zeta_{k} \leq t, \zeta_{i}>\zeta_{k}, \quad i=1, \ldots, k-1, k+1, \ldots r\right\}= \\
& =\int_{D} f_{1}\left(x_{1}\right) f_{2}\left(x_{2}\right) \ldots f_{r}\left(x_{r}\right) d x_{1} d x_{2} \ldots d x_{r}= \\
= & \int_{D} \lambda_{1} e^{-\lambda_{1} x_{1}} \lambda_{2} e^{-\lambda_{2} x_{2}} \ldots \lambda_{r} e^{-\lambda_{r} x_{r}} d x_{1} d x_{2} \ldots d x_{r}
\end{aligned}
$$

$$
D=\left\{\left(x_{1}, x_{2}, \ldots, x_{r}\right): 0 \leq x_{k} \leq t, x_{i}>x_{k} \quad i=1, \ldots, k-1, k+1, \ldots r\right\} .
$$

Finally, we get

$$
Q_{r+1 k}(t)=\frac{\lambda_{k}}{\Lambda}\left(1-e^{-\Lambda t}\right), \quad t \geq 0, \quad \Lambda=\lambda_{1}+\cdots+\lambda_{r} .
$$

Change of the state $k$ to $r+1$ in a time not greater than $t$ takes place if and only if the event $\left\{\gamma_{k} \leq t\right\}$ occurs

Therefore

$$
Q_{k r+1}(t)=P\left\{\gamma_{k} \leq t\right\}=G_{k}(t) .
$$

Finally the kernel of discussed

$$
Q(t)=\left[\begin{array}{llll}
0 & \cdots & 0 & G_{1}(t) \\
0 & \cdots & 0 & G_{2}(t) \\
\cdots & \cdots & 0 & G_{r}(t) \\
0 & \cdots & \frac{\lambda_{r}}{\Lambda}\left(1-e^{-\Lambda t}\right) & 0
\end{array}\right],
$$

where

$$
\Lambda=\lambda_{1}+\cdots+\lambda_{r}
$$


Furthermore, we assume

$$
P\{X(0)=r+1\}=1 .
$$

The matrix of transition probabilities of embedded Markov chain $\left\{X\left(\tau_{n}\right): \quad n=0,1,2, \ldots\right\}$ corresponding to the kernel (17), has the form

$$
P=\left[\begin{array}{llll}
0 & \cdots & 0 & 1 \\
0 & \cdots & 0 & 1 \\
\cdots & & & \\
0 & \cdots & 0 & 1 \\
\frac{\lambda_{1}}{\Lambda} & \cdots & \frac{\lambda_{r}}{\Lambda} & 0
\end{array}\right]
$$

Expected time of return to the "up" state is expectation of the random variable $\Theta_{r+1 r+1}$. From (5) we obtain

In discussed model we have

$$
E\left(\Theta_{r+1 r+1}\right)=E\left(T_{r+1}\right)+\sum_{k=1}^{r} p_{r+1 k} E\left(\Theta_{k r+1}\right) .
$$

$$
E\left(\Theta_{k r+1}\right)=E\left(\gamma_{k}\right), \quad p_{r+1 k}=\frac{\lambda_{k}}{\Lambda}, \quad k=1, \ldots, r, \quad E\left(T_{r+1}\right)=\frac{1}{\Lambda},
$$

Therefore

$$
E\left(\Theta_{r+1 r+1}\right)=\frac{1+\lambda_{1} E\left(\gamma_{1}\right)+\cdots+\lambda_{r} E\left(\gamma_{r}\right)}{\Lambda} .
$$

From (6) we get equation for the second moment of the random variable $\Theta_{r+1 r+1}$.

$$
E\left(\Theta_{r+1 r+1}^{2}\right)=E\left(T_{r+1}^{2}\right)+\sum_{k=1}^{r} p_{r+1 k}\left[2 E\left(T_{r+1 k}\right) E\left(\Theta_{k r+1}\right)+E\left(\Theta_{k r+1}^{2}\right)\right] .
$$

Because

$$
E\left(\Theta_{k r+1}^{2}\right)=E\left(\gamma_{k}^{2}\right), \quad p_{r+1 k} E\left(T_{r+1 k}\right)=\frac{\lambda_{k}}{\Lambda^{2}}, \quad k=1, \ldots, r, \quad E\left(T_{r+1}^{2}\right)=\frac{2}{\Lambda^{2}},
$$

hence

$$
E\left(\Theta_{r+1 r+1}^{2}\right)=\frac{2}{\Lambda^{2}}\left[1+\sum_{k=1}^{r} \lambda_{k} E\left(\gamma_{k}\right)\right]+\frac{1}{\Lambda} \sum_{k=1}^{r} \lambda_{k} E\left(\gamma_{k}^{2}\right) .
$$

Applaing well known formula

$$
D\left(\Theta_{r+1 r+1}\right)=\sqrt{E\left(\Theta_{r+1 r+1}^{2}\right)-\left[E\left(\Theta_{r+1 r+1}\right)\right]^{2}}
$$

We obtain the standard deviation of the random variable $\Theta_{r+1 r+1}$. 
The renewal process generated by return times of semi-Markov process in...

Proces odnowy generowany przez czasy powrotu procesu semi-Markowskiego...

\section{Numerical example}

$$
\begin{gathered}
\text { Let } r=3, \lambda_{1}=0,001\left[\frac{1}{h}\right], \lambda_{2}=0,002\left[\frac{1}{h}\right], \lambda_{3}=0,002\left[\frac{1}{h}\right], \\
E\left(\gamma_{1}\right)=24[h], \quad E\left(\gamma_{2}\right)=32[h], \quad E\left(\gamma_{3}\right)=20[h], \\
\mathrm{E}\left(\gamma_{1}^{2}\right)=640\left[h^{2}\right], \quad \mathrm{E}\left(\gamma_{2}^{2}\right)=1088\left[h^{2}\right], \quad \mathrm{E}\left(\gamma_{3}^{2}\right)=436\left[h^{2}\right] .
\end{gathered}
$$

Because

$E\left(T_{r+1}\right)=\frac{1}{\Lambda}$, where $\Lambda=\lambda_{1}+\lambda_{2}+\lambda_{3}$, then $\Lambda=0,005$ and $E\left(T_{4}\right)=200[\mathrm{~h}]$. Substituting in (21), (22) i (23) the proper numbers we obtain

$E\left(\Theta_{44}\right)=225,6[\mathrm{~h}], \quad E\left(\Theta_{44}^{2}\right)=90265,6\left[\mathrm{~h}^{2}\right], \quad D\left(\Theta_{44}\right)=198,42[\mathrm{~h}]$. Now the formula (10) has the form

$$
m(t)=\frac{t}{E\left(\Theta_{44}\right)}, \sigma(t)=\frac{D\left(\Theta_{44}\right)}{E\left(\Theta_{44}\right)} \sqrt{\frac{t}{E\left(\Theta_{44}\right)}} .
$$

Substituting the proper numbers, for $t=10000$ we obtain $m(10000)=44,33, \sigma(10000)=0,585$. It means that the expected number of the object failures in the time interval $[0,10000][h]$ is approximately equal to 44,33 with the standard deviation 0,585 . From the presented theorem it follows that

$$
P\left(42 \leq V_{44}(10000) \leq 46\right)=\phi\left(\frac{46-44,33}{0,585}\right)-\phi\left(\frac{42-44,33}{0,585}\right),
$$

where $\phi(x), \quad x \in(-\infty, \infty)$ is $\mathrm{CDF}$ of the normal standard distribution. Finally we have

$$
P\left(42 \leq V_{44}(10000) \leq 46\right) \approx 0,998 \text {. }
$$

\section{References}

[1] Cinlar E.:: Markov renewal theory. Adv.Appl. Prob. (1969), 123-187.

[2] Grabski, F.: Semi-markowskie modele niezawodności i eksploatacji. Badania Systemowe, tom30 ; IBS PAN, Warszawa, 2002.

[3] Grabski, F. : Semi-Markov Processes: Application in System Reliability and Maintenance. Elsevier, Amsterdam, Boston, Heidelberg, London, New York Oxford, Paris,San Diego, San Francisco, Sydney,Tokyo, 2015.

[4] Korolyuk VS, Turbin AF. Semi-Markov processes and their applications. Naukova Dumka, Kiev 1976, (in Russian).

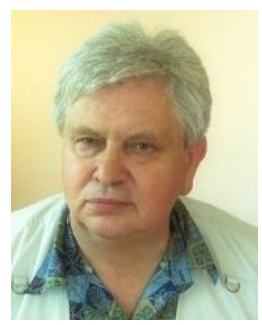

prof. dr hab. Franciszek Grabski Full Professor and Head of Department of Mathematics and Physics of Polish Naval Academy. Professor Grabski's main mathematical research interest focuses on probability theory, in particular its applications in system reliability theory and practice. He has constructed and tested several new reliability stochastic models and developed the Bayesian methods application in reliability. He is author or coauthor more than 120 scientific papers, course-books and monographs in the probability and reliability field. 


\section{PROCES ODNOWY GENEROWANY PRZEZ CZASY POWROTU PROCESU SEMI-MARKOWSKIEGO W MODELACH NIEZAWODNOŚCI}

\section{Wprowadzenie}

Wielkościami związanymi z procesem semi-markowskimi (SM) są między innymi czas pierwszego przejścia procesu do określonego stanu i czas powrotu. Wielkości te są zmiennymi losowymi, których rozkład i parametry można wyznaczyć w oparciu o jądro procesu SM. Kolejne czasy powrotu procesu wraz z czasem pierwszego przejścia pozwalają zdefiniować ogólny proces odnowy. Łącząc wyniki teorii procesów SM oraz teorii odnowy można otrzymać równania pozwalające znaleźć rozkład i parametry tego procesu odnowy a także rozkład graniczny. W pracy zostanie przedstawiony przykład zastosowania przytoczonych pojęć i twierdzeń w semi-markowskich modelu niezawodności.

\section{Niezbędne definicje i twierdzenia}

Zakładamy, że stan $j \in S$ jest stanem powracającym, silnie osiągalnym ze stanu $i \in S$ regularnego procesu semi-markowskiego $\{X(t): t \geq 0\}$ o jądrze typu ciągłego $Q(t)$. Rozważmy ciąg zmiennych losowych

$$
\Theta_{i j}^{(1)}, \Theta_{j j}^{(2)}, \Theta_{j j}^{(3)}, \ldots
$$

gdzie $\Theta_{i j}^{(1)}=\Theta_{i j}$ oznacza chwilę pierwszego osiągnięcia stanu $j \in S$ przez proces startujący w chwili 0 ze stanu $i \in S$, natomiast zmienne losowe $\Theta_{j j}^{(n)}, \quad n=2,3, \ldots$ oznaczają długości przedziałów czasu, po których następują kolejne powroty do stanu $j \in S$. Zmienna losowa $\Theta_{i j}^{(1)}$ ma rozkład o dystrybuancie

$$
\Phi_{i j}(t)=P\left\{\Theta_{i j}^{(1)} \leq t\right\},
$$

Można wykazać [ ], że dystrybuaty $\Phi_{i j}(t), i, j \in \mathrm{S}$, przy założeniach, które są spełnione w rozważanych tu modelach, spełniają układ równań całkowych, który poddany transformacji Laplace'a-Stieltjesa przyjmuje postać

$$
\tilde{\phi}_{i A}(s)=\sum_{j \in A} \tilde{q}_{i j}(s)+\sum_{k \in A^{\prime}} \tilde{q}_{i k}(s) \tilde{\phi}_{k A}(s),
$$

gdzie

$$
\tilde{\phi}_{i A}(s)=\int_{0}^{\infty} e^{-s t} d \Phi_{i A}(t), \quad \tilde{q}_{i j}(s)=\int_{0}^{\infty} e^{-s t} d Q_{i j}(t) .
$$


The renewal process generated by return times of semi-Markov process in... Proces odnowy generowany przez czasy powrotu procesu semi-Markowskiego...

Otrzymaliśmy układ równań liniowych, w których współczynnikami są transformaty $\tilde{q}_{i j}(s)$, a niewiadomymi transformaty $\tilde{\phi}_{i A}(s)$. Układ ten jest równoważny równaniu macierzowemu

$$
\left(I-q_{A^{\prime}}(s)\right) \phi_{A^{\prime}}(s)=b(s)
$$

gdzie $I=\left[\delta_{i j}: i, j \in A^{\prime}\right] \quad$ jest macierzą jednostkową, $q_{A^{\prime}}(s)=\left[\tilde{q}_{i j}(s): i, j \in A^{\prime}\right]$ jest podmacierzą kwadratową macierzy transformat $q(s)$, natomiast macierze

$$
\phi_{A^{\prime}}(s)=\left[\tilde{\phi}_{i A}(s): i \in A^{\prime}\right]^{T}, \quad b(s)=\left[\sum_{j \in A} \tilde{q}_{i j}(s): i \in A^{\prime}\right]^{T}
$$

są jednokolumnowymi macierzami odpowiednich transformat Laplace'a-Stieltjesa. Układ ten pozwala znaleźć równania dla momentów zmiennych losowych $\Theta_{i A}, i \in A^{\prime}$. Wartości oczekiwane $E\left(\Theta_{i A}\right), i \in A^{\prime}$ są jedynymi rozwiązaniami układu równań

gdzie

$$
\left(I-P_{A^{\prime}}\right) \bar{\Theta}_{A^{\prime}}=\bar{T}_{A^{\prime}}
$$

$$
P_{A^{\prime}}=\left[p_{i j}: i, j \in A^{\prime}\right], \bar{\Theta}_{A^{\prime}}=\left[E\left(\Theta_{i A}\right): i \in A^{\prime}\right]^{T}, \quad \bar{T}_{A^{\prime}}=\left[E\left(T_{i}\right): i \in A^{\prime}\right],
$$

Drugie momenty są jedynymi rozwiązaniami układu równań liniowych, który w notacji macierzowej ma postać

$$
\begin{gathered}
\left(I-P_{A^{\prime}}\right) \bar{\Theta}_{A^{\prime}}^{2}=B_{A}, \\
P_{A^{\prime}}=\left[p_{i j}: i, j \in A^{\prime}\right], \quad \bar{\Theta}_{A^{\prime}}=\left[E\left(\Theta_{i A}^{2}\right): i \in A^{\prime}\right]^{T}, \quad B_{A}=\left[b_{i A}: i \in A^{\prime}\right]^{T}, \\
b_{i A}=E\left(T_{i}^{2}\right)+2 \sum_{k \in A^{\prime}} p_{i k} E\left(T_{i k}\right) E\left(\Theta_{k A}\right) .
\end{gathered}
$$

oraz $I$ jest macierzą jednostkową.$$
\text { Zmienne losowe } \Theta_{j j}^{(n)}, \ldots, n=2,3, \ldots \text { oznaczające kolejne czasy powrotu mają }
$$
jednakowy rozkład określony przez dystrybuantę

$$
\Phi_{j j}(t)=P\left\{\Theta_{j j}^{(n)} \leq t\right\}=P\left\{\Theta_{j j} \leq t\right\} .
$$

Równanie dla transformat Laplace'a-Stieltjesa ma postać

$$
\tilde{\phi}_{j j}(s)=\tilde{q}_{j j}(s)+\sum_{k \in S-\{j\}} \tilde{q}_{j k}(s) \tilde{\phi}_{k j}(s),
$$

a równania dla wartości oczekiwanych i momentów zwykłych drugiego rzędu są natępujące;

$$
\begin{gathered}
E\left(\Theta_{j j}\right)=E\left(T_{j}\right)+\sum_{k \in S-\{j\}} p_{j k} E\left(\Theta_{k j}\right), \\
E\left(\Theta_{j j}^{2}\right)=E\left(T_{j}^{2}\right)+\sum_{k \in S-\{j\}} p_{j k} E\left(\Theta_{k j}^{2}\right)+2 \sum_{k \in S-\{j\}} p_{j k} E\left(T_{j k}\right) E\left(\Theta_{k j}\right) .
\end{gathered}
$$


Można wykazać, że zmienne losowe

$$
\Theta_{i j}^{(1)}, \Theta_{j j}^{(2)}, \ldots, \Theta_{j j}^{(n)}, \quad n=2,3, \ldots
$$

są wzajemnie niezależne.

Niech

$$
S_{i j}^{(n)}=\Theta_{i j}^{(1)}+\Theta_{j j}^{(2)}+\cdots+\Theta_{j j}^{(n)}, \quad n=2,3, \ldots
$$

oraz

$$
V_{i j}(t)=\sup \left\{n: S_{i j}^{(n)} \leq t\right\}, \quad t \geq 0,
$$

Wartość zmiennej losowej $V_{i j}(t)$ oznacza liczbę „wejść" procesu semiMarkowskiego do stanu $j \in S \mathrm{w}$ przedziale [0,t]. Proces losowy $\left\{V_{i j}(t): t \geq 0\right\}$ nazywamy procesem odnowy generowanym przez czasy powrotu procesu semiMarkowskiego.

Adaptując twierdzenia klasycznej teorii odnowy możemy sformułować twierdzenie o asymptotycznym rozkładzie procesu losowego $\left\{V_{i j}(t): t \geq 0\right\}$ przy $t \rightarrow \infty$.

Twierdzenie [ 2 ], [ 3 ]. Jeżeli stan j jest stanem powracajacym, silnie osiagalnym ze stanu $i \in S$ oraz zmienne losowe $\Theta_{i j}, \Theta_{j j}$ maja skończone dodatnie wartości oczekiwane i skończone dodatnie wariancje, to

$$
\lim _{t \rightarrow \infty} P\left\{\frac{V_{i j}(t)-\frac{t-E\left(\Theta_{i j}\right)+E\left(\Theta_{j j}\right)}{E\left(\Theta_{j j}\right)}}{\sqrt{\frac{V\left(\Theta_{j j}\right) t}{\left.E\left(\Theta_{j j}\right)\right]^{3}}}} \leq x\right\}=\frac{1}{\sqrt{2 \pi}} \int_{-\infty}^{x} e^{\frac{u^{2}}{2}} d u .
$$

$\mathrm{Z}$ twierdzenia tego wynika, że dla dużego $t$ zmienna losowa $V_{i j}(t)$ ma w przybliżeniu rozkład normalny $N(m(t), \sigma(t))$ o wartości oczekiwanej i odchyleniu standardowym

$$
m(t)=\frac{t-E\left(\Theta_{i j}\right)+E\left(\Theta_{j j}\right)}{E\left(\Theta_{j j}\right)}, \sigma(t)=\sqrt{\frac{D^{2}\left(\Theta_{j j}\right) t}{\left[E\left(\Theta_{j j}\right)\right]^{3}}} .
$$

Parametry $E\left(\Theta_{i j}\right), E\left(\Theta_{j j}\right), E\left(\Theta_{j j}^{2}\right)$ możemy łatwo obliczyć w oparciu o równania (3), (5), (6) .Wariancję obliczamy korzystając z dobrze znanego związku

$$
D^{2}(\Theta)=E\left(\Theta_{j j}^{2}\right)-\left[E\left(\Theta_{j j}\right)\right]^{2} .
$$


The renewal process generated by return times of semi-Markov process in... Proces odnowy generowany przez czasy powrotu procesu semi-Markowskiego...

\section{SM-model odnawialnego systemu o strukturze szeregowej}

Przedstawimy semi-markowski model odnawialnego systemu o szeregowej strukturze niezawodności, zakładając wykładniczy rozkład czasu zdatności elementów oraz dowolny rozkład czasu odnowy elementów. Tego rodzju sytem był modelowany i badany $\mathrm{w}$ pracach [1], [2], [3] . W tej pracy omówimy jedną $\mathrm{z}$ charakteryk niezawodnościowych tego systemu - liczbę uszkodzeń $j$-tego rodzaju w przedziale czasu $[0, t]$.

\section{Opis i zalożenia}

System o szeregowej strukturze niezawodności składa się $\mathrm{z} r$ elementów. Zakładamy, że czas zdatności elementu o numerze $k$ jest nieujemną zmienną losową $\zeta$ o rozkładzie wykładniczym określonym przez gęstość

$$
f_{k}(t)=\lambda e^{-\lambda t} I_{[0, \infty)}(t) .
$$

Ze struktury niezawodnościowej systemu wynika, że uszkodzenie użytkowanego systemu następuje w chwili uszkodzenia jakiegokolwiek elementu. Uszkodzony element jest odnawiany. Przyjmujemy, że czas odnowy $k$-tego elementu jest nieujemną zmienną losową $\gamma_{k}$ o rozkładzie określonym przez dystrybuantę

$$
G_{k}(t)=P\left\{\gamma_{k} \leq t\right\} .
$$

Jak wiemy, rozkład wykładniczy charakteryzuje się własnością braku pamięci. Wynika stąd, że odnowa elementu jest jednocześnie odnową systemu. Przyjmujemy, że kolejne czasy zdatności oraz kolejne czasy odnowy elementu o numerze $k$ są niezależnymi kopiami zmiennych losowych $\zeta_{k}$ oraz $\gamma_{k}$ odpowiednio. Zakładamy, że zmienne losowe $\zeta_{1}, \ldots, \zeta_{r}, \gamma_{1}, \ldots, \gamma_{r}$ są wzajemnie niezależne. Przyjmujemy, że zmienne losowe $\gamma_{1}, \ldots, \gamma_{r}$ mają dodatnie wartości oczekiwane i skończone, dodatnie wariancje.

\section{Model}

Przyjmujemy następujące stany procesu:

$r+1$ - użytkowanie zdatnego systemu,

$k$ - odnowa elementu o numerze $k, k=1, \ldots r$.

Zbiór stanów niezdatności ma postać $A=\{1,2, \ldots, r\}$, natomiast zbiór stanów zdatności jest jednoelementowy $A^{\prime}=\{r+1\}$.

Niech $0=\tau_{0}<\tau_{1}<\cdots<\tau_{n} \cdots$ oznaczają chwile $\mathrm{w}$ których następuje zmiana stanu systemu. Chwile te są chwilami awarii systemu lub chwilami, w których rozpoczyna się użytkowanie zdatnego systemu.

$\mathrm{Z}$ przyjętych założeń wynika, że stan systemu $\mathrm{w}$ chwili $\tau_{n+1}$ oraz czas trwania stanu osiągniętego $\mathrm{w}$ chwili $\tau_{n}$ nie zależy od stanów przyjętych $\mathrm{w}$ chwilach $\tau_{0}, \tau_{1}, \ldots, \tau_{n-1} \ldots$ oraz czasów ich trwania. Proces stochastyczny $\{X(t): t \geq 0\}$ określony wzorem

$$
X(t)=k \quad \text { dla } \quad k \in\left[\tau_{n}, \tau_{n+1}\right), \quad k \in S=\{1, \ldots, r, r+1\}
$$

jest więc procesem semi-Markowa. 
$\mathrm{Z}$ postaci opisu fukcjonowania obiektu w sensie niezawodności wynika postać jądra procesu.

$$
Q(t)=\left[\begin{array}{llll}
0 & 0 & \cdots & Q_{1 r+1}(t) \\
0 & 0 & \cdots & Q_{2 r+1}(t) \\
\cdots & & & \\
0 & 0 & \cdots & Q_{r r+1}(t) \\
Q_{r+11}(t) & Q_{r+12}(t) & \cdots & 0
\end{array}\right]
$$

Model zostanie skonstruowany, gdy określimy wszystkie elementy jądra tego procesu SM. Określimy najpierw elementy ostatniego wiersza macierzy $Q(t)$. Zmiana stanu z $r+1$ na $k,(k=1, \ldots, r)$ w czasie nie większym niż $t$ następuje wtedy i tylko wtedy, gdy zachodzi zdarzenie

$$
\left\{0 \leq \zeta_{k} \leq t, \zeta_{i}>\zeta_{k} \geq 0, \quad i=1, \ldots, k-1, k+1, \ldots r\right\} .
$$

Stąd, wykorzystując niezależność czasów zdatności mamy

$$
\begin{aligned}
Q_{r+1, k}(t) & =P\left\{\zeta_{k} \leq t, \zeta_{i}>\zeta_{k}, \quad i=1, \ldots, k-1, k+1, \ldots r\right\}= \\
& =\int_{D} f_{1}\left(x_{1}\right) f_{2}\left(x_{2}\right) \ldots f_{r}\left(x_{r}\right) d x_{1} d x_{2} \ldots d x_{r}= \\
& =\int_{D} \lambda_{1} e^{-\lambda_{1} x_{1}} \lambda_{2} e^{-\lambda_{2} x_{2}} \ldots \lambda_{r} e^{-\lambda_{r} x_{r}} d x_{1} d x_{2} \ldots d x_{r}
\end{aligned}
$$

gdzie

$$
D=\left\{\left(x_{1}, x_{2}, \ldots, x_{r}\right): 0 \leq x_{k} \leq t, x_{i}>x_{k} \quad i=1, \ldots, k-1, k+1, \ldots r\right\} .
$$

Zamieniając całkę po zbiorze $D$ na całkę iterowaną otrzymujemy

$$
Q_{r+1 k}(t)=\frac{\lambda_{k}}{\Lambda}\left(1-e^{-\Lambda t}\right), \quad t \geq 0, \quad \Lambda=\lambda_{1}+\cdots+\lambda_{r}
$$

Zmiana stanu z $k$ na $r+1 \mathrm{w}$ czasie nie większym niż $t$ ma miejsce wtedy i tylko wtedy, gdy zachodzi zdarzenie $\left\{\gamma_{k} \leq t\right\}$. Zatem

$$
Q_{k r+1}(t)=P\left\{\gamma_{k} \leq t\right\}=G_{k}(t)
$$


The renewal process generated by return times of semi-Markov process in... Proces odnowy generowany przez czasy powrotu procesu semi-Markowskiego...

Ostatecznie otrzymujemy następującą postać jądra procesu

$$
Q(t)=\left[\begin{array}{llll}
0 & \cdots & 0 & G_{1}(t) \\
0 & \cdots & 0 & G_{2}(t) \\
\cdots & \cdots & 0 & G_{r}(t) \\
0 & \cdots & \frac{\lambda_{r}}{\Lambda}\left(1-e^{-\Lambda t}\right) & 0
\end{array}\right]
$$

gdzie

$$
\Lambda=\lambda_{1}+\cdots+\lambda_{r}
$$

Zakładamy dodatkowo, że

$$
P\{X(0)=r+1\}=1 .
$$

Macierz prawdopodobieństw przejść włożonego łańcucha Markowa

ma postać:

$$
\left\{X\left(\tau_{n}\right): \quad n=0,1,2, \ldots\right\}
$$

$$
P=\left[\begin{array}{llll}
0 & \cdots & 0 & 1 \\
0 & \cdots & 0 & 1 \\
\cdots & & & \\
0 & \cdots & 0 & 1 \\
\frac{\lambda_{1}}{\Lambda} & \cdots & \frac{\lambda_{r}}{\Lambda} & 0
\end{array}\right]
$$

Oczekiwany czas powrotu do stanu zdatności jest wartością oczekiwaną zmiennej losowej $\Theta_{r+1 r+1}$. Ze wzoru (5) wynika równanie

$$
E\left(\Theta_{r+1 r+1}\right)=E\left(T_{r+1}\right)+\sum_{k=1}^{r} p_{r+1 k} E\left(\Theta_{k r+1}\right) .
$$

Ponieważ w rozważanym modelu

$$
E\left(\Theta_{k r+1}\right)=E\left(\gamma_{k}\right), \quad p_{r+1 k}=\frac{\lambda_{k}}{\Lambda}, \quad k=1, \ldots, r, \quad E\left(T_{r+1}\right)=\frac{1}{\Lambda},
$$

więc

$$
E\left(\Theta_{r+1 r+1}\right)=\frac{1+\lambda_{1} E\left(\gamma_{1}\right)+\cdots+\lambda_{r} E\left(\gamma_{r}\right)}{\Lambda} .
$$

Ze wzoru otrzymujemy równanie dla drugiego momentu czasu powrotu do stanu zdatności.

$$
E\left(\Theta_{r+1 r+1}^{2}\right)=E\left(T_{r+1}^{2}\right)+\sum_{k=1}^{r} p_{r+1 k}\left[2 E\left(T_{r+1 k}\right) E\left(\Theta_{k r+1}\right)+E\left(\Theta_{k r+1}^{2}\right)\right]
$$


Ponieważ

$E\left(\Theta_{k r+1}^{2}\right)=E\left(\gamma_{k}^{2}\right), \quad p_{r+1 k} E\left(T_{r+1 k}\right)=\frac{\lambda_{k}}{\Lambda^{2}}, \quad k=1, \ldots, r, \quad E\left(T_{r+1}^{2}\right)=\frac{2}{\Lambda^{2}}$,

więc

$$
E\left(\Theta_{r+1 r+1}^{2}\right)=\frac{2}{\Lambda^{2}}\left[1+\sum_{k=1}^{r} \lambda_{k} E\left(\gamma_{k}\right)\right]+\frac{1}{\Lambda} \sum_{k=1}^{r} \lambda_{k} E\left(\gamma_{k}^{2}\right) .
$$

Korzystając ze wzoru

$$
D\left(\Theta_{r+1 r+1}\right)=\sqrt{E\left(\Theta_{r+1 r+1}^{2}\right)-\left[E\left(\Theta_{r+1 r+1}\right)\right]^{2}}
$$

otrzymujemy odchylenie standardowe zmiennej losowej $\Theta_{r+1} r+1$.

\section{Przykład numeryczny}

Przyjmujemy

$$
\begin{array}{ccl}
r=3, \lambda_{1}=0,001\left[\frac{1}{h}\right], \lambda_{2}=0,002\left[\frac{1}{h}\right], & \lambda_{3}=0,002\left[\frac{1}{h}\right], \\
E\left(\gamma_{1}\right)=24[h], & E\left(\gamma_{2}\right)=32[h], & E\left(\gamma_{3}\right)=20[h], \\
\mathrm{E}\left(\gamma_{1}^{2}\right)=640\left[h^{2}\right], & \mathrm{E}\left(\gamma_{2}^{2}\right)=1088\left[h^{2}\right], & \mathrm{E}\left(\gamma_{3}^{2}\right)=436\left[h^{2}\right] .
\end{array}
$$

Ponieważ $E\left(T_{r+1}\right)=\frac{1}{\Lambda}, \quad$ gdzie $\Lambda=\lambda_{1}+\lambda_{2}+\lambda_{3}$, więc $\Lambda=0,005$ oraz $E\left(T_{4}\right)=200[\mathrm{~h}]$. Podstawiając do wzorów (21), (22) i (23) wartości liczbowe otrzymujemy

$E\left(\Theta_{44}\right)=225,6[\mathrm{~h}], \quad E\left(\Theta_{44}^{2}\right)=90265,6\left[\mathrm{~h}^{2}\right], \quad D\left(\Theta_{44}\right)=198,42$ [h]. Wzór (10) przyjmuje teraz postać

$$
m(t)=\frac{t}{E\left(\Theta_{44}\right)}, \sigma(t)=\frac{D\left(\Theta_{44}\right)}{E\left(\Theta_{44}\right)} \sqrt{\frac{t}{E\left(\Theta_{44}\right)}} .
$$

Podstawiając liczby dla $t=10000$ otrzymujemy $m(10000)=44,33$, $\sigma(10000)=0,585$ co oznacza, że oczekiwana liczba uszkodzeń urządzenia $\mathrm{w}$ przedziale $[0,10000][h]$ jest $\mathrm{w}$ przybliżeniu równa $44,33 \mathrm{z}$ odchyleniem standardowym 0,585 . Z tezy przytoczonego twierdzenia wynika, że

$$
P\left(42 \leq V_{44}(10000) \leq 46\right)=\phi\left(\frac{46-44,33}{0,585}\right)-\phi\left(\frac{42-44,33}{0,585}\right),
$$

gdzie $\phi(x), \quad x \in(-\infty, \infty)$ jest dystrybuantą standardowego rozkładu normalnego.

Ostatecznie otrzymujemy

$$
P\left(42 \leq V_{44}(10000) \leq 46\right) \approx 0,998 .
$$


The renewal process generated by return times of semi-Markov process in...

Proces odnowy generowany przez czasy powrotu procesu semi-Markowskiego...

\section{Literatura}

[1] Cinlar E.:: Markov renewal theory. Adv.Appl. Prob. (1969), 123-187.

[2] Grabski, F.: Semi-markowskie modele niezawodności i eksploatacji. Badania Systemowe, tom30 ; IBS PAN, Warszawa, 2002.

[3] Grabski, F. : Semi-Markov Processes: Application in System Reliability and Maintenance. Elsevier, Amsterdam, Boston, Heidelberg, London, New York Oxford, Paris,San Diego, San Francisco, Sydney,Tokyo, 2015.

[4] Korolyuk VS, Turbin AF. Semi-Markov processes and their applications. Naukova Dumka, Kiev 1976, (in Russian).

Profesor dr hab. Franciszek Grabski jest Kierownikiem Katedry Matematyki i Fizyki Akademii Marynarki Wojennej. Głównym nurtem jego zainteresowań naukowych jest probabilistyka, a szczególnie jej zastosowania w teorii niezawodności. Zajmowat sie konstruowaniem $i$ badaniem stochastycznych modeli niezawodności $i$ eksploatacji oraz metodami bayesowskimi w niezawodności. Jest autorem ponad 120 prac naukowych, a także autorem lub współautorem skryptów, podręczników i książek. 